\title{
Search for High Energy Neutron Point Sources with IceTop
}

\author{
The IceCube Collaboration, \\ ${ }^{\dagger}$ http://icecube.wisc.edu/collaboration/authors/icrc15_icecube \\ E-mail: sutherland.54@osu.edu
}

\begin{abstract}
IceTop can detect an astrophysical flux of neutrons from Galactic sources as an excess of cosmic ray air showers arriving from the source direction. Neutrons are undeflected by the Galactic magnetic field and can typically travel $10(E / \mathrm{PeV}) \mathrm{pc}$ before decay. Two searches through the IceTop dataset are performed to look for a statistically significant excess of events with energies above $10 \mathrm{PeV}\left(10^{16} \mathrm{eV}\right)$ arriving within a small solid angle. The all-sky search method covers from $-90^{\circ}$ to approximately $-50^{\circ}$ in declination. A targeted search is also performed, looking for significant correlation with candidate sources in different target sets. Flux upper limits can be set in both searches.
\end{abstract}

Corresponding authors: Michael S. Sutherland ${ }^{1 *}$,

${ }^{1}$ Department of Physics and the Center for Cosmology and Astro-Particle Physics,

The Ohio State University,

Columbus, Ohio 43210, USA

The 34th International Cosmic Ray Conference,

30 July- 6 August, 2015

The Hague, The Netherlands

\footnotetext{
${ }^{*}$ Speaker.
} 


\section{Introduction}

Secondary neutral particles are an expected signature of hadronic acceleration in Galactic sources. These particles would be produced as the cosmic ray primaries interact within the dense environment surrounding their source [1]. A source of high energy neutrons would manifest as a point source in cosmic ray arrival directions since neutrons are not deflected by magnetic fields. Because of the energy-dependent neutron lifetime, the search is sensitive to sources within a distance of $\approx 10 E_{P e V}$ parsecs to Earth. Since plausible accelerators are no closer than tens of pc, searches at energies above $10 \mathrm{PeV}$ are the most promising.

The Pierre Auger Observatory recently performed a search $[2,3]$ at ultra-high energies (> $\mathrm{EeV}$ ) finding no significant signal excesses or correlations with catalogs of Galactic objects and establishing flux upper limits. KASCADE [4] and CASA-MIA [5] found no point sources in the northern sky, also resulting in flux limits. AGASA [6] and a re-analysis [7] of SUGAR data reported excesses towards the Galactic center, although these were later not confirmed by the Pierre Auger analyses.

In this analysis, we perform two searches using four years of IceTop data: one search for general hotspots on the sky and another search for correlations with nearby Galactic sources. This latter analysis is not optimized to identify specific types of neutral particles. A dedicated photon search will be reported later.

\section{IceTop Reconstructions and Dataset}

IceTop [8], located $2835 \mathrm{~m}$ above sea level, is the surface air shower array of the IceCube Neutrino Observatory at the geographical South Pole. Its current configuration consists of 81 stations spread over an area covering $1 \mathrm{~km}^{2}$ with an average station separation of $125 \mathrm{~m}$. A single station is comprised of a pair of tanks filled with frozen water and containing two photomultipliers detectors each. Detector construction started in 2005 and finished in 2010. Cosmic ray reconstruction relies on the optical detection of Cherenkov radiation in the tanks emitted by secondary particles produced in high energy interactions in the upper atmosphere. IceTop reconstruction uses information from individual tanks, including position, deposited charge, and pulse timing, to infer the air shower direction, core location, and shower size estimate $S_{125}$ [9]. The relationship between $S_{125}$ and cosmic ray energy is determined by comparison with Monte Carlo simulations.

Snow accumulates on top of the stations with time which attenuates the electromagnetic portion of the shower and lowers the shower size estimate $S_{125}$. Snow depth measurements for each tank are performed twice a year so that the depth at the time of an event can be interpolated. A correction factor is applied during event reconstruction so that the resulting $S_{125}$ distributions for each year are consistent. The correction is an exponential function described by an attenuation length that differs for each year.

In this analysis, events are selected by requiring good fits to the shower lateral distribution and reconstructed core locations lying within $400 \mathrm{~m}$ of the array center (not near the boundary). A zenith angle cut of $37^{\circ}$ and an energy threshold of $10 \mathrm{PeV}$ and $100 \mathrm{PeV}$ for the all-sky and targeted search, respectively, are applied. 1233487 (12558) events pass these cuts for each analysis, 
respectively, from a total livetime of 3.76 years. The average angular resolution above $10 \mathrm{PeV}$ is approximately $0.5^{\circ}$.

\section{Search Methods}

For both search methods, top-hat ${ }^{1}$ search windows are drawn around areas of interest on the sky. In the all-sky search, these windows are centered on the pixels of a high-resolution HEALPix [10] map whereas in the targeted search they are centered on the catalog objects. This map contains 19800 points within the IceTop field-of-view from which to draw the search windows with a typical spacing of $0.6^{\circ}$. The radius of the search window $\left(0.52^{\circ}\right)$ is based on the actual IceTop point-spread function and is chosen such that it optimizes the sensitivity to a point source. The data is first binned using the pixels of a higher resolution HEALPix map ("bin map") than the search window map. The content of search window $i$ is the sum of contents of those pixels in the bin map whose centers fall within the search window and is labelled $n_{i}\left(b_{i}\right)$ for the dataset (background).

The background expectation for each search window is determined by time-scrambling the dataset many times. For each time and zenith angle pair in the data, a random azimuthal angle is chosen between 0 and $2 \pi$. This preserves the time and zenith distributions. The background bin map is taken as the average of the maps for the time-scrambled datasets. $10^{4}\left(10^{6}\right)$ scrambled sets are generated for the all-sky (targeted) search.

The significance of signal excesses are computed differently for each method. For the allsky search, the significance for each window is determined using the Li-Ma method [11], where we take the Li-Ma parameters $N_{o n}=n$ and $N_{o f f}=b / \alpha$. The parameter $\alpha$ is taken to be the $b / \xi$, where $\xi$ is the sum of the contents of search windows within $\pm 90^{\circ}$ in right ascension of the window of interest. This definition of $\alpha$ provides a local estimate of $N_{\text {off }}$ for each search window, since IceTop observes a large-scale anisotropy [12]. For the targeted search, we calculate the Poisson probability $p_{i}$ for observing $n_{i}$ or more events within the search window expecting $b_{i}$ for each object. Fisher's method [13] combines a set of independent probabilities to determine a single measure of significance for the set. A supplemental measure of significance is provided by Good's method [14] which allows for weights to be assigned to each probability. Here, these weights are proportional to the object's recorded electromagnetic flux, its exposure to IceTop, and the expected flux attenuation factor ${ }^{2}$ resulting from neutron decay. The weights are normalized such that the sum is 1 for each target set. Treating the Fisher and Good probabilities $\left(P_{F}, P_{G}\right)$ as individual test statistics, we calculate the fraction of time-scrambled datasets with corresponding values less than that observed with the data. This post-trials fraction is an unbiased indicator of the correlation probability between the dataset and each source set.

The targeted search is performed on three classes of candidate sources: millisecond pulsars [15], $\gamma$-ray pulsars [16], and high mass X-ray binaries [17] (HMXB). Distances for each candidate are cross-checked with the TeVCat catalog [18] and objects must lie within the zenith angle cut. Sources that appear in multiple sets are retained only in the smaller set, resulting in 17 objects in the $\gamma$-ray pulsar set, 16 objects in the msec pulsar set, and 20 objects in the HMXB set. The

\footnotetext{
${ }^{1}$ Selecting events using a hard cut on the space angle between the event direction and the window center.

${ }^{2}$ The survival probability for neutrons from the distance of the candidate source assuming a $E^{-2}$ energy spectrum.
} 
location of each source object are shown in Figure 1. The Galactic plane is depicted by a green band to illustrate the preferential association of the $\gamma$-ray pulsar and HMXB catalogs with that part of the sky.

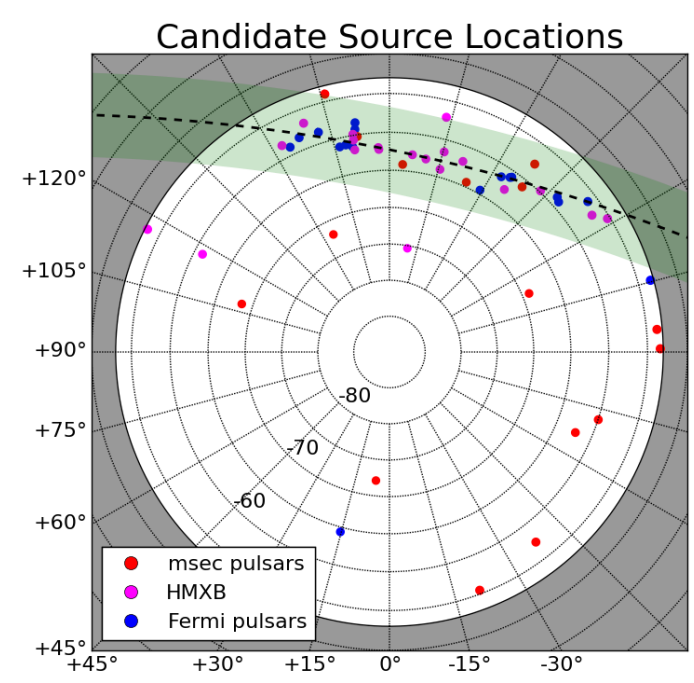

Figure 1: Equatorial polar skymap of each catalog set. The dashed black line indicates the Galactic plane and the green band shows roughly $\pm 5^{\circ}$. Each circle is $0.5^{\circ}$ in radius.

Flux upper limits are calculated using $F_{U L}=1.39 s_{U L} /(T A \cos \theta \varepsilon)$, where $s_{U L}$ is the upper limit on the expected number of signal events, $T$ is the livetime, $A \cos \theta$ is the geometrical area exposed to the search window which depends on the zenith angle, and $\varepsilon$ is the reconstruction efficiency (taken as 95\%). The factor 1.39 is a compensation factor resulting from correcting $s_{U L}$ for signal events that fall outside the search window, since the window radius is based on the angular resolution. The flux limit is a time-averaged value based on the IceTop exposure. Particularly for the objects in the targeted source sets, transient fluxes may temporarily exceed these limits.

\section{Results}

Figure 2 shows the differential (left) and cumulative (right) distribution of Li-Ma values compared to the isotropic expectation. In both images, the blue (green) line shows the Li-Ma significance distribution for the data (isotropy). The dashed line shows the Gaussian function expected if deviations from isotropy are due only to statistical fluctuations. The gray shaded region in the cumulative plot shows the 95\% containment band for isotropy; the presence of search windows with statistically significant signal excess would extend above and to the right of this band. The absence of such a feature indicates that no statistically significant signal excess is observed. Figure 3 shows skymaps of the Li-Ma and flux upper limit values for each search window. No statistically significant clustering on the sky is observed, particularly along the Galactic plane depicted by the black dotted $\left(b=0^{\circ}\right)$ and solid $\left( \pm 2.5^{\circ}\right)$ lines. 
Figure 4a shows the mean flux upper limit as a function of declination. The limits are strongest near the South Pole due to the maximal exposure, but there is greater uncertainty since there are fewer search windows.
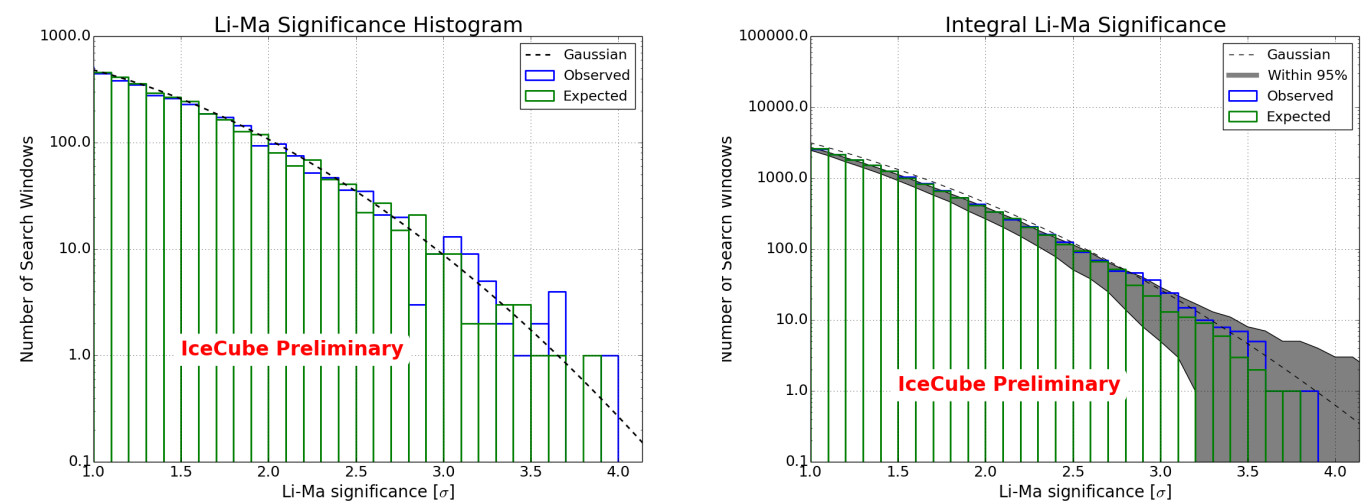

Figure 2: Histograms of Li-Ma values (blue) and the isotropic expectation (green).
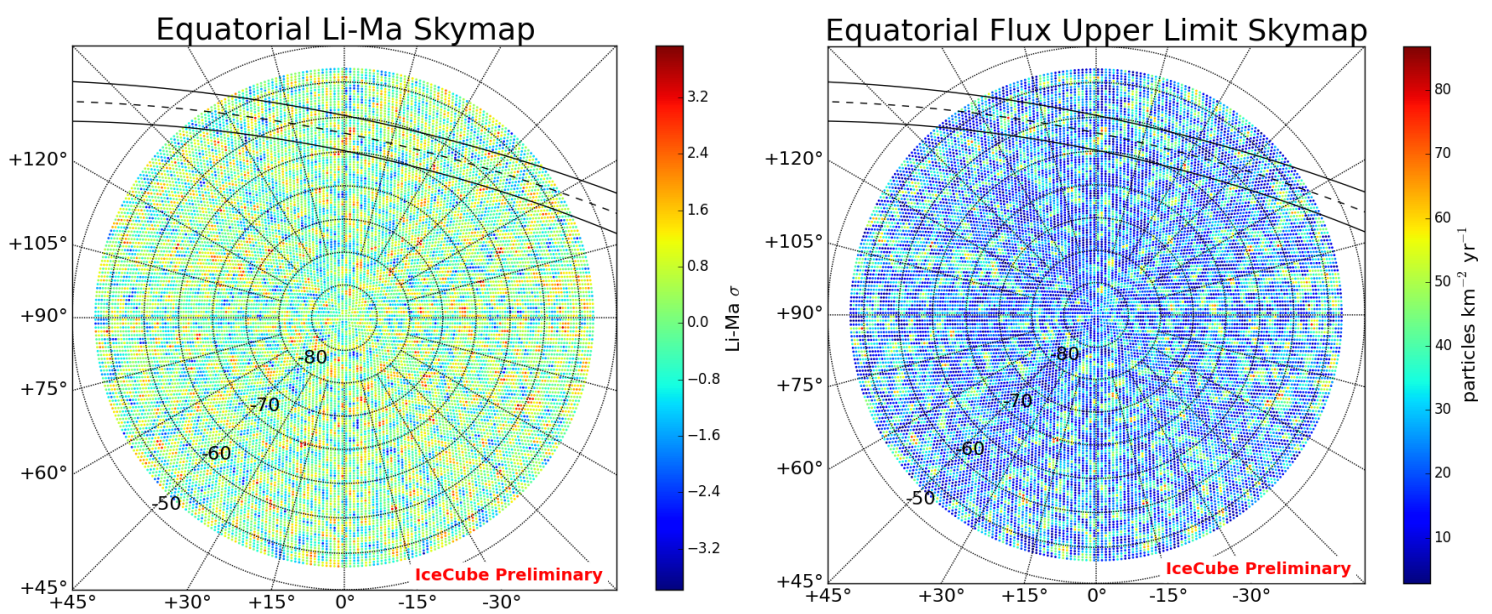

Figure 3: Equatorial polar skymap of Li-Ma (left) and flux upper limit (right) values for each search window. The solid black lines depict a $5^{\circ}$ band centered on the Galactic plane.

Table 1 lists $P_{F}$ and $P_{G}$ for each catalog. The quantity in parentheses shows the post-trials probability value. No significant correlation is observed with any catalog. Table 2 lists details of the object with the smallest Poisson $p$ in each catalog, including the neutron flux $F_{U L}$ and energy flux $F_{U L}^{E}$ for an $E^{-2}$ spectrum. The post-trials probability (in parentheses) for the minimum $p$ in each catalog also indicates that no evidence for PeV neutron flux from the candidates is observed.

There exists an underfluctuation in the data along $b=0^{\circ}$ compared to the background expectation, as illustrated in Figure $4 \mathrm{~b}$. The preferential clustering of the $\gamma$-ray pulsar and HMXB catalogs along the Galactic plane combined with this underfluctuation results in artificially high $P_{F}$ and $P_{G}$. This behavior is verified by rotating the catalogs in right ascension. We also note that 4 pairs of 


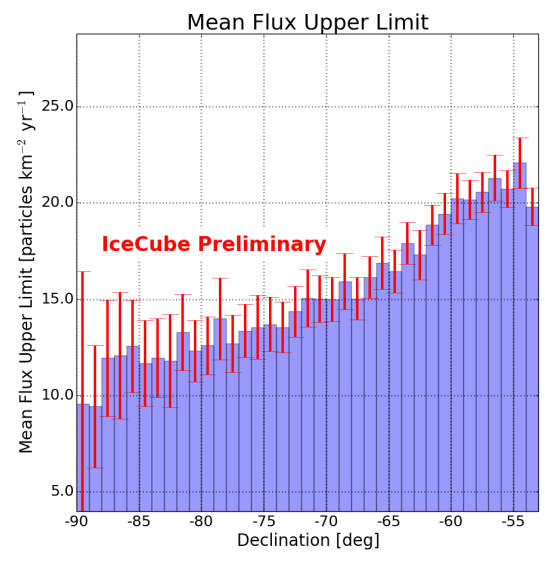

(a) Mean flux upper limit for $1^{\circ}$ declination bins.

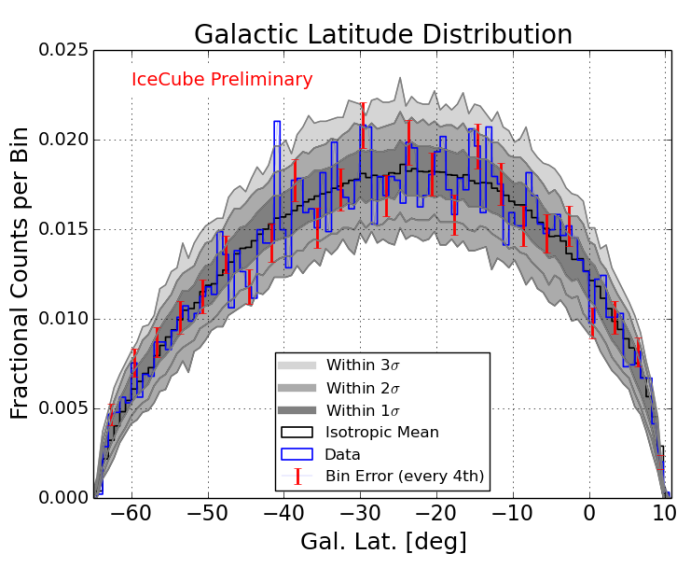

(b) Distribution of number of events above $100 \mathrm{PeV}$ as a function of Galactic latitude.

Figure 4: Left: The error bars indicate the statistical uncertainty on the mean value. The uncertainties are larger at small zenith angles since there are less search windows than at larger zenith angles. Right: The blue histogram shows the data; the black line shows the isotropic expectation from 800 time-scrambled datasets. The red error bars show the Poisson uncertainty in the data histogram and are plotted only for every 4th bin to reduce clutter. The gray shaded bands depict the $68 \%, 95 \%$, and $99 \%$ containment bands for isotropy in each latitude bin.

\begin{tabular}{|c||c|c|}
\hline Catalog & $P_{F}$ & $P_{G}$ \\
\hline \hline$\gamma$-ray & $0.998(0.947)$ & $0.887(0.727)$ \\
\hline msec & $0.820(0.457)$ & $0.898(0.792)$ \\
\hline HMXB & $0.999(0.997)$ & $0.945(0.973)$ \\
\hline
\end{tabular}

Table 1: Targeted search results with each catalog. First column lists the catalog, second column lists the Fisher probability $P_{F}$ and post-trials value, and third column lists the Good probability $P_{G}$ and its post-trials value (in parentheses).

objects, where objects in each pair are distinct and from different catalogs, lie within $1^{\circ}$ of each other. Results are consistent with Table 1 when we mask the object with the farther distance.

\section{Summary}

IceTop does not observe a statistically significant point source of cosmic ray arrival directions in an all-sky search within the field-of-view. Additionally, no significant correlation is found with known Galactic objects thought to be capable producing PeV neutrons. The mean flux upper limits for individual declination bands correspond to energy fluxes between about $1.2-1.5 \mathrm{eV} \mathrm{cm}^{-2}$ $\mathrm{sec}^{-1}$, which are comparable to $\mathrm{TeV} \gamma$ fluxes for Galactic objects [19]. Photons and neutrons can be produced through pion production of protons with ambient photons and nuclei. Photons resulting from $\pi^{0}$ decay take a small fraction of the proton energy. Neutrons result from charge-exchange interactions where a $\pi^{+}$emerges with the proton's positive charge and the neutron retains most of 


\begin{tabular}{|c||c|c|c|c|c|c|c|c|}
\hline Catalog & Object Name & R.A. [deg] & Dec. [deg] & $n$ & $b$ & $F_{U L}\left[\mathrm{~km}^{-2} \mathrm{yr}^{-1}\right]$ & $F_{U L}^{E}\left[\mathrm{eV} \mathrm{cm}^{-2} \mathrm{sec}^{-1}\right]$ & $p_{i}$ \\
\hline \hline$\gamma$-ray & J1048-5832 & 162.05 & -58.53 & 5 & 2.40 & 11.35 & 0.65 & $0.095(0.665)$ \\
\hline msec & J1933-6211 & 293.39 & -62.20 & 6 & 2.57 & 14.87 & 0.86 & $0.047(0.419)$ \\
\hline HMXB & 2 S1417-624 & 215.30 & -62.70 & 4 & 2.65 & 10.11 & 0.58 & $0.274(0.993)$ \\
\hline
\end{tabular}

Table 2: Targeted search results for most significant object in each catalog. Columns lists the catalog name, object name, equatorial coordinates, number of events $n$ and background expectation $b$ within the search window, neutron number $F_{U L}$ and energy $F_{U L}^{E}$ flux upper limits, and Poisson p-value $p_{i}(n, b)$ and post-trials value (in parentheses).

the energy. For parent protons with an $E^{-2}$ spectrum, these upper limits provide constraints on the production origin of the $\mathrm{TeV} \gamma_{\mathrm{s}}$ and the energy evolution of proton spectra.

The non-observation of $\mathrm{PeV}$ neutron sources may simply indicate that such sources were not active during the data-taking period. Sources may emit particle jets continuously, but their number may be few and the jets are not oriented towards the Earth. Additionally, environments around any sources may not be dense enough to facilitate neutron production. These possibilities would apply if the parent proton spectra extended beyond tens of PeV, which may not be the case. IceTop observes a cosmic ray flux that becomes progressively heavier with energy and a decreasing proton fraction [20], which is about $20 \%$ at $10 \mathrm{PeV}$. Neutrons may be produced at lower energies where protons comprise a larger fraction, but the resulting lower energies severely limit neutron observations to near the production sites.

\section{References}

[1] R. M. Crocker et al., Astrophys. J. 622 (2005) 892.

[2] Pierre Auger Collaboration, A. Aab et al., Astrophys. J. 760 (2012) 148.

[3] Pierre Auger Collaboration, A. Aab et al., Astrophys. J. 789 (2014) L34.

[4] KASCADE Collaboration, T. Antoni et al., Astrophys. J. 608 (2004) 865.

[5] CASA-MIA Collaboration, J. Cronin et al., Phys. Rev. D D45 (1992) 4835.

[6] AGASA Collaboration, N. Hayashida et al., Astropart. Phys. 10 (1999) 303.

[7] J. Bellido et al., Astropart. Phys. 15 (2001) 167.

[8] IceCube Collaboration, R. Abbasi et al., Nucl. Instrum. Meth. A700 (2013) 188.

[9] IceCube Collaboration, M. Aartsen et al., Phys. Rev. D 4 (2013) 042004.

[10] K. Gorski et al., Astrophys. J. 622 (2005) 759.

[11] T. Li and Y. Ma, Astrophys. J. 272 (1983) 317.

[12] IceCube Collaboration, PoS (ICRC2015) 334 these proceedings.

[13] R. Fisher, Statistical Methods for Research Workers, Edinburgh: Oliver and Boyd (1925).

[14] I. Good, JRSS B 17 (1955) 264.

[15] R. Manchester et al., Astrophys. J. 129 (2005) 1993. 
[16] Fermi Collaboration, A. Abdo et al., Astrophys. J. Suppl. 208 (2013) 17.

[17] Q. Liu et al., A\&A 455 (2007) 1165.

[18] The TeVCat catalog, http://tevcat.uchicago.edu.

[19] J. Hinton et al., ARA\&A 47 (2009) 523.

[20] IceCube Collaboration, PoS (ICRC2015) 274 these proceedings. 